\title{
The Impacts of Exercise-Intervention on the Prevention and Treatment of Some Types of Cancer
}

\author{
Han Kyo Seo PhD \\ Department of Beauty Health, Shinhan University, Uijeongbu, Korea
}

PURPOSE: Cancer is a disease affecting millions of people and one of the primary causes of death worldwide like cardiovascular diseases. Nonpharmacological and nonoperative methods such as proper exercise-intervention, nutrition, maintaining the activity of daily living (ADL), psychological methods can also improve the physical function, cancer progression, and health-related quality of life (HRQOL). However it is extremely important, little light shed on the impacts of exercise-intervention on cancer prevention and treatments. Therefore, the purpose of this study was to analyze the impacts of nonpharmaceutical approaches such as diverse exercise-intervention on cancer prevention and treatments with psychological and physiological health for cancer patients. Moreover, this paper wants to combine knowledge regarding the many experimental papers and systemic reviews of cancer researches and exercise intervention liter-ature.

METHODS: A meta-analysis and systemic reviews of the impacts of an exercise intervention on cancer prevention and treatments were conducted to the PRISMA guidelines. 20 research articles were selected for final inclusion and extraction. And electronic analysis such as PubMed, Google scholar was carried for this study up to May 2020. The search keyword was 'exercise-intervention and cancer'.

RESULTS: We can suggest that physical exercise-intervention might be a suitable combination partner to exercise-related immune therapy in the prevention, delay, and treatment of cancer patients. Moreover, physical exercise-intervention directly enhances the QOL, ADL, healthy life, and even resilience of cancer patients.

CONCLUSIONS: Therefore, these exercise interventions can be effective at reducing cancer symptoms, and enhancing cancer treatments for diverse and many cancer patients. Reversing low muscle mass can enhance cancer therapy outcomes and mortality. Nutrition strategies also might be helpful. A Follow-up study about specific high-quality tailoring exercise-intervention program on counteracting cancer symptoms, cancer prevention and treatment, physiological and psychological health and HRQOL is urgently needed in this area.

Key words: Exercise-intervention, Cancer prevention, Treatment, Cancer patients

\section{INTRODUCTION}

Physical exercise has been well documented on physical fitness, functional capacity, cardiovascular disease, reduced risk of mortality and even cancer prevention.

Recent experimental research revealed that voluntary exercise leads to a significant reduction in tumor size and tumor growth across several murine models [1].

Increased immune cell functions and the influx of immune cell as well as changes of body composition, systemic inflammation have been suggested to a significant role. Different type and different mode of exercise interventions have been analyzed in the caner populations and have resulted in general recommendations for increasing physical activity.

These kinds of physical activity contributes to enhanced to health and utilitarian outcomes in cancer populations irrespective of the cancer type and stroma.

Exercise interventions at moderate and vigorous intensity are reported to safe and evaluated in fitness improvement such as $\mathrm{VO} 2$ max, $\mathrm{VO} 2$ peak and muscle strength compare to at a lower level of exercise intensity [2]. Lower exercise intensity also revealed the enhancement of health-re-

Corresponding author: Han Kyo Seo Tel +82-10-5309-7171 Fax +82-31-870-3449 E-mail hkseo001dgmail.com

Received 1 Jul 2020 Revised 30 Dec 2020 Accepted 31 Jan 2021

(a)This is an Open Access article distributed under the terms of the Creative Commons Attribution Non-Commercial License (https://creativecommons.org/licenses/by-nc/4.0/) which permits unrestricted non-commercial use, distribution, and reproduction in any medium, provided the original work is properly cited. 
lated quality of life (HRQOL), fatigue, and depression in cancer populations.

Numerous experimental and review researches underlined the benefits of exercise interventions during active cancer treatment with positive effect on several side effects [3-5]. Generally, exercise augments in stroke volume and cardiac output to meet oxygen demands, and further changes of blow flow. Therefore, metabolic rate and metabolism goes up.

Endocrine system also involved in incorporating the physiological responses during rest and physical exercise. Catecholamine hormones are reported to significantly increased during exercise from the adrenal glands by affecting metabolic process and immune system. Therefore, these hormone activates the heart rate, blood glucose, metabolic rates and immune responses. Catecholamine-mediated direct impacts of exercise on cancer cell research was already performed in Copenhagen team among breast cancers [5].

Exercise-induced mobilization of NK cells is supposedly induced by an increase in catecholamines. NK cell mobilization is achieved after 30 min of physical activity. This normally function to clear virus infected and transduced cells [1]. Therefore, this kinds of hormones can be important factor in the physical exercise associated enhanced tumor control by several lymphocytes. Exercise-mediated myokines such as IL-6 activate the mobilization of NK cells and immune system.

It was well documented the physical exercise intervention impacted on favorable effects irrespective of the exercise timing (pre-treatment, during cancer treatment, and post treatment). However, the timing of exercise-intervention can be the important factor of cancer type and cancer adjuvant treatment period [2]. These treatments highlights the enormously positive effects on depression, anxiety, sleep pattern, physical conditions and cancer-related fatigue [2].

Resistance training increased muscle mass, NK cell and NKT cell function of TNF-ft [6] of breast cancer patients. Moderate-intensity exercise improved NK cell immune recovery for hematopoietic stem cell transplantation pediatric patients [7].

One of the research about the connection between immunity and aging revealed that immunosenescence is caused by reduced cytotoxic ability of NK cells, thymus atrophy, reduced naïve T cell, and declined B cell function [8].

Moreover, physical inactivity and unhealthy metabolic conditions were connected with the decreased NK cell function. Regular physical exercise improves immune responses and reverses the effect of immunosenescence with aging. This may be due to the systemic inflammation reduction, stress reduction, enhanced regulatory $\mathrm{T}$ cell function, and increased immunosurveillance $[9,10]$. Therefore, physical exercise can be a powerfully practical intervention for elderly people and patients living with some type of cancer.

Cancer is a disease affecting millions of people and one of the primary causes of death worldwide like cardiovascular diseases. Nonpharmacological and nonoperative methods such as proper exercise-intervention, nutrition, maintaining activates of daily living (ADL), psychological methods can also improve the physical function, cancer progression, and HRQOL [11].

However, although it is extremely important, little light shed on the impacts of exercise intervention on cancer prevention and treatments. Therefore, the purpose of this study was to analyze the impacts of nonpharmaceutical approach such as diverse exercise-intervention on cancer prevention and treatments with psychological and physiological health for cancer patients. And moreover, this paper want to combine knowledge regarding the many experimental papers and systemic reviews of cancer researches and exercise intervention literature.

\section{METHODS}

A meta-analysis and systemic reviews of the impacts of exercise intervention on cancer prevention and treatments were conducted with regard to the PRISMA guidelines. 20 research articles were selected for final inclusion and extraction.

And electronic analysis such as PubMed, Google scholar was carried for this study up to May, 2020. The research title was 'exercise-intervention and cancer'.

Cancer is a disease affecting millions of people and one of the primary causes of death worldwide like cardiovascular diseases. Nonpharmacological and nonoperative methods such as proper exercise-intervention, nutrition, maintaining activates of daily living (ADL), psychological methods can also improve the physical function, cancer progression, and HRQOL [11].

One of the important research about exercise and cancer reported that an intrinsic flaw in the cytotoxic capacity of NK cells turned up in sedentary elderly people but not in active elderly people who execute daily physical activity [12].

There is a home-based exercise intervention research for stomach cancer patients receiving oral chemotherapy, In this study, 8 weeks of homebased intervention impacts on cancer related fatigue, anxiety, QOL, and 
NK cell ratio for stomach cancer patients receiving oral chemotherapy after surgery [13]. 8 weeks of exercise education and home- based exercise were performed for this patients. The QoL and NK ratio of exercised group increased while that of the control group decreased. It highlights that home- based exercise program is safe and practical treatments for cancer patients during chemotherapy.

This was explained by the another research results of habitually active elderly people have much higher NK cells cytotoxic activities [12]. Generally, $51 \mathrm{Cr}$ release assay, IL-12 and INF- $f$ assay can demonstrate the NK cell activity.

Many research revealed that an association of lower NK cell activity with an increased risk of cancer development. Leptin, adiponectin from excessive body fat mass affect systemic inflammation and immune systems.

It was well known that NK cells are the first line of defense against malignant cells and NK cell functions are severely impaired with sedentary life and obesity. Impaired NK cell functions were evaluated with diet-induced obese rats.

However, NK cell function was reactivated by the 3 months of nutrition and exercise combined programs [14]. Bariatric surgery, diet and physical activity were reactivated NK cell function.

It might be the cause of exercise-induced INF- $f$ and IL-15 signaling in NK cell cancer immunotherapy. Immunotherapy has emerged over the past more than 10 years next to chemotherapy, radiation, and surgery $[14,15]$. INF- $f$ also upregulates the function and differentiation of immune cells such as NK cells and T cells.

Circulating leptin, inflammatory cytokines, adipokines were closely involved with the cancer risks and it can be reversed by the regular physical exercise.

The cancer cells killing mechanisms by NK cells was the uptake of tumor antigens by dendritic cells and the induction of tumor-specific $\mathrm{T}$ cell responses [16,17].

Activated NK cells express the $f$-adrenergic receptors, which was induced by the epinephrine by physical exercise. NK cells also substantial role for the anti-cancer effects in the absence case of T cells, B cells and dendritic cells responses. Therefore, it can be possible of early cancer cells surveillance, while T cells, B cells and dendritic cells play an important role against established tumor [1]. Exercise-mediated positive alteration in body mass index, hormonal status, systemic inflammation have been suggested to play an important role. Interleukine-6 (IL-6) is well noted to be pro-inflammatory myokine of chronic inflammation and bacterial infections, which is released by contracting muscles [1]. Released IL-6 also activates NK cells mobilization and immune activation with enhanced level of epinephrine as well as AMPK signaling and cellular metabolism.

There were many research efforts were undertaken about 'exercise and cancer' researches. The harvest of T cells was possible by physical exercise prior to surgery.

It also enhance the T cells function before surgery. Muscle mass is also an important role in cancer patients. Muscle is an important tissue for normal ADL status of cancer patients. It is the site of myokine production and strength, mobility, balance, and movement is urgently needed to cancer patients by the combined help of physical exercise and protein supplements [17]. Optimal energy intake and regular physical exerciseintervention were needed not only avoid weight loss but also to maintain muscle mass by stimulating protein synthesis and suppressing protein breakdown $[18,19]$.

It was well reported that many cancer patients experience poor nutritional status, which lethally effects clinical outcomes. Poor nutritional status is primarily manifested by severe muscle mass depletion $[11,18]$ whereas only $10 \%$ of cancer patients are underweight.

Low muscle mass is common phenomenon irrespective of the stage of cancer [11]. It is also an health predictor such as cancer progression and surgical complications as well as ADL and physical function status.

Therefore, reversing low muscle mass combined by continuous physical exercise and protein supplements can enhance the symptom, mobility, progression, even mortality.

Proper protein supplementation (1.0-1.5 g/kg/day, 15-20\% of total calory intake) is an important for maintaining muscle mass because of its anabolic stimulus [17].

Prostate and melanoma showed physical exercise associated with higher risks of cancer in the exercised people, whereas physical exerciseintervention is associated with lower risks of many cancer types.

One of the systemic reviews of exercise and cancer literature reveals that exercise intervention are well established tool as beneficial and safe for many cancer patients and this also contributes to enhanced functional outcomes and health of cancer population. However, this research suggest that therapeutic exercise is additionally recommended as a rehabilitative approach for cancer patients experiencing more specific functional disability [2].

Resistance training has a beneficial impacts on the NK and natural killer T cell (NKT) expression of TNF-ft indicating useful tools of im- 
proving inflammatory profile in breast cancer survivors [6]. Moderate -intensity exercise program was also impacts on the NK cell immune recovery in pediatric patients after hematopoietic stem cell transplantation [7].

Even single bout of physical exercise has 'immuno-enhancing' effects and has been used to efficiently enhance vaccine responses in cancer patients. This might be due to the inflammation reduction, thymic mass maintenance, immune enhancement and improved immunosurveillance (by Treg cell) [10].

We can suggest that physical exercise-intervention might be a suitable combination partner to immune therapy in the prevention, delay and treatment of cancer patients. Moreover, physical exercise-intervention directly enhance the QoL, ADL, healthy life and even resilience of cancer patients.

Therefore, these exercise interventions can be effective at reducing cancer symptoms, and cancer treatments for diverse and many cancer patients. Reversing low muscle mass has the possible to enhance cancer therapy outcomes and mortality [11]. Nutrition strategies also might be helpful.

Follow-up study about dietary intervention through the intake of zinc, glutamine, $n-3$ polyunsaturated fatty acids and specific high-quality tailoring exercise-intervention program on counteracting cancer symptoms, cancer prevention and treatment, physiological and psychological health and QoL is urgently needed in this area.

\section{CONCLUSIONS}

This study analyzed physical exercise-intervention might be a suitable combination partner to immune therapy in the prevention, delay and treatment of cancer patients. Moreover, physical exercise-intervention directly enhance the QoL, ADL, healthy life and even resilience of cancer patients. Therefore, these exercise interventions can be effective at reducing cancer symptoms, and cancer treatments for diverse and many cancer patients. Reversing low muscle mass has the possible to enhance cancer therapy outcomes and mortality. Nutrition strategies also might be helpful.

Follow-up study about dietary intervention through the intake of zinc, glutamine, $n-3$ polyunsaturated fatty acids and specific high-quality tailoring exercise-intervention program on counteracting cancer symptoms, cancer prevention and treatment, physiological and psychological health and QoL is urgently needed in this area.

\section{CONFLICT OF INTEREST}

The authors declare that they do not have conflict of interest.

\section{AUTHOR CONTRIBUTION}

Conceptualization, Writing, Review \& Editing: H. Seo.

\section{ORCID}

Han Kyo Seo https://orcid.org/0000-0001-7074-7810

\section{REFERENCES}

1. Idorn M, Straten P. Exercise and cancer: from "healthy" to "therapeutic"?. Cancer Immunol Immunother. 2017;66:667-71.

2. Nicole L, Jennifer B, Anne S, Kerri M, Judith W. A systematic review of exercise systematic reviews in the cancer literature (2005-2017). PM R 2017;9:S347-S384.

3. Dittus K, Toth M, Priest J, O'Brien P, Kokinda N, et al. Effects of an exercise-based oncology rehabilitation program and age on strength and physical function in cancer survivors. Support Care Cancer. 2020; 28(8):3747-54

4. Morales JS, Valenzuela PL, Herrera-Olivares AM, Bano-Rodrigo A, Castillo-Garcia A, et al. Exercise Interventions and Cardiovascular Health in Childhood Cancer: a meta-analysis. Int J Sports Med. 2020 Epub ahead of print.

5. Medysky ME, Temesi J, Culos-Reed SN, Millet GY. Exercise, sleep and cancer-related fatigue: are they related? Neurophysiol Clin. 2017;47(2): 111-22.

6. Dethlefsen C, Hansen LS, Lillelund C, Andersen C, Gehl J, et al. Exercise-induced catecholamines activate the Hippo tumor suppressor pathway to reduce risks of breast cancer development. Cancer Res. 2017;77:4894-904.

7. Hagstrom AD, Marshall PW, Lonsdale C, Papalia S, Cheema BS, et al. The effect of resistance training on markers of immune function and inflammation in previously sedentary women recovering from breast cancer: a randomized controlled trial. Breast Cancer Res Treat. 2016; 155(3):471-82

8. Chamorro-vina C, Valentin J, Fernandez L, Gonzalez, Vicent M, et al. Influence of a moderate-intensity exercise program on early NK cell 
immune recovery in pediatric patients after reduced-intensity hematopoietic stem cell transplantation. Integr Cancer Ther. 2017;16(4):46472.

9. Buford TW, Willoughby DS. Impact of DHEA(S) and cortisol on immune function in aging: a brief review. Appl Physiol Nutr Metab. 2008;33(3):429-33.

10. Jung YS, Park JH, Park DI, Sohn CI, Lee JM, et al. Physical inactivity and unhealthy metabolic status are associated with decreased natural killer cell activity. Yonsei Med J. 2018; 59(4):554-62.

11. Simpson RJ, Kunz H, Agha N, Graff R. Exercise and the regulation of immune functions. Prog Mol Biol Transl Sci. 2015;135:355-80.

12. Prado CM, Purcell SA, Laviano A. Nutrition interventions to treat low muscle mass in cancer. J Cachexia Sarcopenia Muscle. 2020;Epub ahead of print.

13. Ogawa K, Oka J, Yamakawa J, Higuchi M. A single bout of exercise influences natural killer cells in elderly women, especially those who are habitually active. J Strength Cond Res. 2005;19(1):45-50.

14. Choi JY, Kang HS. Effects of a home-based exercise program for pa- tients with stomach cancer receiving oral chemotherapy after surgery. J Korean Acad Nurs. 2012;42(1):95-104.

15. Jahn J, Spielau M, Brandsch C, Stangl GI, Delank KS, et al. Decreased NK cell functions in obesity can be reactivated by fat mass reduction. Obesity. 2015;23(11):2233-41.

16. Rautela J, Huntington ND. IL-15 signaling in NK cell cancer immunotherapy. Curr Opin Immunol. 2017;44:1-6.

17. Jung NC, Lee JH, Chung KH, Kwak YS, Lim DS. Dendritic cell-based immunotherapy for solid tumors. Transl Oncol. 2018;11:686-90.

18. Ryan AM, Power DG, Daly L, Cushen SJ, Ni Bhuachalla E, et al. Cancer-associated malnutrition, cachexia and sarcopenia: the skeleton in the hospital closet 40 years later. Proc Nutr Soc. 2016;75;(2):1-13.

19. Purcell SA, Elliott SA, Baracos VE, Chu QSC, Prado CM. Key determinants of energy expenditure in cancer and implications for clinical practice. Eur J Clin Nutr. 2016;70:1230-8.

20. Patel DI, Abuchowski K, Sheikh B, Rivas P, Musi N, et al. Exercise preserves muscle mass and force in a prostate cancer mouse model. Eur J Transl Myol. 2019;29(4):8520. 